\title{
Computer-Aided Diagnosis of Pulmonary Nodules Using Three-Dimensional Thoracic CT Images
}

\author{
Y. Kawata ${ }^{1}$, N. Niki ${ }^{1}$, H. Ohmatsu ${ }^{2}$, M. Kusumoto ${ }^{3}$, R. Kakinuma ${ }^{2}$, \\ K. Mori ${ }^{4}$, H. Nishiyama ${ }^{5}$, K. Eguchi ${ }^{6}$, M. Kaneko ${ }^{3}$, N. Moriyama ${ }^{3}$ \\ ${ }^{1}$ Dept. of Optical Science, Univ. of Tokushima, ${ }^{2}$ National Cancer Center East, \\ ${ }^{3}$ National Cancer Center, ${ }^{4}$ Tochigi Cancer Center, \\ ${ }^{5}$ The Social Health Medical Center, ${ }^{6}$ National Shikoku Cancer Center \\ \{kawata,niki\}@opt.tokushima-u.ac.jp
}

\begin{abstract}
We are developing computerized feature extraction and classification methods to analyze malignant and benign pulmonary nodules in threedimensional (3-D) thoracic CT images. Internal structure features were derived from CT density and 3-D curvatures to characterize the inhomogeneous of CT density distribution inside the nodule. In the classification step, we combined an unsupervised $\mathrm{k}$-means clustering (KMC) procedure and a supervised linear discriminate (LD) classifier. The KMC procedure classified the sample nodules into two classes by using the mean CT density values for two different regions such as a core region and a complement of the core region in 3-D nodule image. The LD classifier was designed for each class by using internal structure features. The stepwise procedure was used to select the best feature subset from multi-dimensional feature spaces. The discriminant scores output from the classifier were analyzed by receiver operating characteristic (ROC) method and the classification accuracy was quantified by the area, $A z$, under the ROC curve. We analyzed a data set of pulmonary nodules in this study. The results of this study indicate the potential of combining the KMC procedure and the LD classifier for computer-aided classification of pulmonary nodules.
\end{abstract}

\section{Introduction}

We are developing computerized feature extraction and classification methods to analyze malignant and benign pulmonary nodules in three-dimensional (3-D) thoracic images. The purpose of this research is to design a hybrid classifier combining an unsupervised and a supervised model to improve the classification performance.

\section{Method and Result}

3-D thoracic images were reconstructed from thin-section CT images obtained by the helical CT scanner. In the 3-D thoracic image, pulmonary nodules were segmented by a deformable surface model. From the resulting nodule region, curvature indexes (shape index and curvedness) and CT density were computed and then a set of 


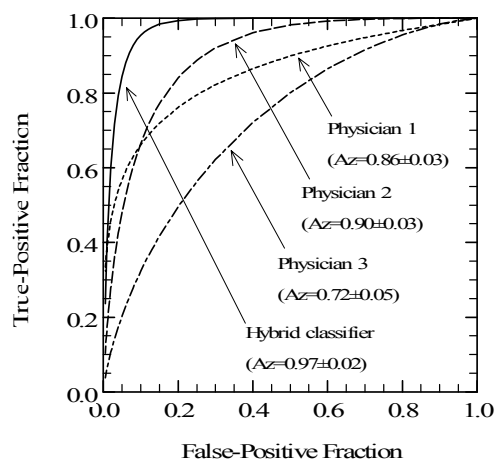

Fig. 1. Comparison of ROC curves of the hybrid classifier and physicians.

histogram features was measured from the distribution of the curvature indexes and CT density over the nodule region. The histogram features measure the amount of voxel which has a particular shape category and CT value in the nodule. Additionally, we introduced the topological and shape distribution features to characterize the internal structure of the nodule. We divided the inside of the nodule into four shape categories by using the shape index value and then, computed the topological features of each 3-D cluster which constructed from a set of voxels with the same shape category. The topological features used here were the Euler number, the number of connected components, cavities, and holes of each 3-D cluster. The shape distribution feature quantified how each shape category distributes inside the nodule by using a technique computing 3-D Euclidean distance transform. In the classification step, we designed a hybrid classifier that combined an unsupervised k-means clustering (KMC) procedure and a supervised linear discriminate (LD) classifier. The KMC procedure classified the sample nodules into two classes by using the mean CT density values for two different regions such as a core region and a complement of the core region in 3-D nodule image. The LD classifier was designed for each class. The discriminate scores output from the classifier were analyzed by receiver operating characteristic (ROC) methodology. The ROC curves using two experienced physicians and one inexperienced physician were shown in Fig.1. The data set included 141 pulmonary nodules (34 benign and 107 malignant cases. The ROC curve of the hybrid classifier was also plotted. The physicians 1, 2, and 3 respectively have 15 years, 12 years, and one year of experience in the chest radiology. This result highlights the promise of the hybrid classifier in the classification between benign and malignant nodules.

\section{Conclusion}

The application results of our method to the $3 \mathrm{D}$ thoracic images have demonstrated that the hybrid classifier is a promising approach for improving the accuracy of classifiers for CAD applications. 